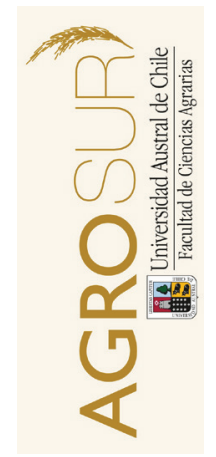

\title{
Efecto de la aplicación de tres estrategias de mejoramiento de praderas degradadas sobre las propiedades hidráulicas del suelo
}

\author{
Effect of the application of three strategies to improve degraded pastures \\ on the hydraulic properties of the soil
}

\author{
Navarro, G. ${ }^{a}$, Balocchi, 0. ${ }^{c}$, López, I. ${ }^{d}$, Clunes, J. ${ }^{a}$, Dörner, J. ${ }^{a b *}$ \\ ${ }^{a}$ Instituto de Ingeniería Agraria y Suelos, Universidad Austral de Chile. Casilla 567, Valdivia, Chile. \\ ${ }^{b}$ Centro de Investigación en Suelos Volcánicos, Universidad Austral de Chile. Casilla 567, Valdivia, Chile. \\ ${ }^{c}$ Instituto de Producción Animal, Universidad Austral de Chile. Casilla 567, Valdivia, Chile. \\ ${ }^{d}$ Institute of Agriculture and Environment, Massey University, Palmerston North, New Zealand.
}

\begin{tabular}{l} 
A R T I C L E I N F O \\
\hline Keywords: \\
Grazing management \\
Soil physical properties \\
Pasture improvement \\
management \\
Degraded pastures \\
\hline Original Research Article, \\
Soil Science
\end{tabular}

${ }^{*}$ Corresponding author:

José Dörner

E-mail address:

josedorner@uach.cl

\begin{abstract}
A B S T R A C T
Southern Chile is characterized by the use of degraded naturalized pastures for livestock production, affecting the physical characteristics of the soil that sustains them. The objective of this study was to determine the effect of three strategies to improve degraded pastures under intensive grazing for milk production on soil hydraulic properties. The pastures were established on Trumao soil (Duric Hapludand), and 4 treatments were carried out (control degraded meadow (DsF), degraded meadow + fertilizers (DcF), planted pasture of Lolium perenne L. (Lp) and Trifolium repens $\mathrm{L}$. (Tr) + fertilizers $(\mathrm{ScF})$ and a mixed sown meadow of L. perenne (Lp), T. repens L. (Tr), Dactylis glomerata L. (Dg), Bromus valdivianus Phil. (Bv) and Holcus lanatus L. (Hl) + fertilizers (McF)), distributed in three complete blocks at random. Disturbed and undisturbed soil samples were taken from each plot and the water retention curves $(\mathrm{pF})$ and pore-size distribution of the treatments were determined, as well as the hydraulic conductivity in saturated phase (Ks), the air permeability (Ka), the stability of the aggregates and the contact angle, and associated chemical parameters. The results obtained show only statistically significant differences in soil air capacity (PDR). The rest of the evaluated parameters did not show significant differences regarding the application of strategies to improve pasture, only trends, mainly due to the short time elapsed between the implementation of the trial and the measurements (2 years).
\end{abstract}

\section{RESUMEN}

La zona sur de Chile se caracteriza por el uso de praderas naturalizadas degradadas en la producción ganadera afectando las características físicas del suelo que la sustenta. El objetivo de este estudio fue determinar el efecto de tres estrategias de mejoramiento de praderas degradadas bajo pastoreos intensivos de producción de leche sobre propiedades hidráulicas del suelo. Se establecieron sobre un suelo Trumao (Duric Hapludand), 4 tratamientos (pradera degradada testigo (DsF), pradera degradada + fertilizantes (DcF), pradera sembrada de Lolium perenne L. (Lp) y Trifolium repens L. (Tr) + fertilizantes (ScF) y una pradera sembrada mixta de L. perenne (Lp), T. repens (Tr), Dactylis glomerata L. (Dg), Bromus valdivianus Phil. (Bv) y Holcus lanatus $\mathrm{L} .(\mathrm{Hl})+$ fertilizantes $(\mathrm{McF}))$, distribuidos en tres bloques completos al azar. De cada parcela se tomaron muestras disturbadas y no disturbadas de suelo y se determinaron las curvas de retención de agua (pF) y distribución de tamaño de poros de los tratamientos, la conductividad hidráulica en fase saturada (Ks), la permeabilidad de aire (Ka), la estabilidad de los agregados y el ángulo de contacto, además de parámetros químicos asociados. Los resultados obtenidos sólo muestran diferencias estadísticamente significativas en la capacidad de aire del suelo (PDR). El resto de los parámetros evaluados no mostraron diferencias significativas con la aplicación de distintas estrategias de mejoramiento de praderas debido principalmente al poco tiempo transcurrido entre la implementación del ensayo y las mediciones (2 años).

Palabras clave: Manejo de pastoreo, propiedades físicas del suelo, mejoramiento de praderas, pradera degradada.

\section{INTRODUCCIÓN}

La condiciones edáficas y climáticas en la zona sur de Chile determinan que una parte importante de la producción pecuaria se caracteriza por el uso de praderas naturalizadas degradadas con predominio de especies gramíneas en su composición (Teuber, 1996). Los principales factores de degradación en las praderas naturalizadas son el sobrepastoreo, fertilización deficiente, condiciones climáticas y labores culturales de conservación de forraje inadecuadas (Balocchi, 2007). Mientras una pradera naturalizada degradada alcanza niveles de 
producción menores a 5 Ton MS ha ${ }^{-1}$ año $0^{-1}$ una de alta producción debería superar las 12 Ton $\mathrm{MS} \mathrm{ha}^{-1}$ año ${ }^{-1}$ bajo condiciones de secano (Seibald et al., 2000).

El pastoreo continuo es perjudicial sobre la pradera cuando su tránsito compacta el suelo, disminuyendo la aireación e infiltración de agua y alterando el balance natural entre especies por su selectividad (Dec et al., 2011, 2012). La compactación del suelo ocurre cuando es sobrepasada su capacidad de soporte, generando una redistribución del volumen poroso (Hartge y Horn, 1991; Hillel, 1998). El tránsito de ganado con una alta carga animal, particularmente, en condiciones de alto contenido volumétrico de agua en el suelo, puede incrementar su densidad aparente. Lo anterior, implica una reducción del volumen de poros y de su continuidad, provocando un desarrollo superficial y poco difuso del sistema radical, lo que disminuye la eficiencia de absorción de agua y nutrientes del perfil de suelo (Allende, 1993; Ellies, 1994; Drewry, 2006). Esta compactación está asociada con fenómenos de deformación interna en el suelo, que ocurren a expensas del sistema poroso y se traducen en una pérdida de calidad del suelo (Cuevas et al., 2004). De esta manera, es fundamental comprender la magnitud del efecto del pastoreo sobre las propiedades físicas del suelo, ya que según la intensidad y la duración del evento de pastoreo pueden afectar el crecimiento de la pradera y su composición (Greenwood y McKenzie, 2001; Taboada, 2007).

La permeabilidad de aire (Ka) y la conductividad hidráulica saturada (Ks) son parámetros de intensidad de los suelos que describen aspectos dinámicos en el tiempo y el espacio (Horn y Kutilek, 2009), relacionados con la funcionalidad del sistema poroso, la distribución, continuidad y tortuosidad de los poros del suelo, siendo sensibles a cambios estructurales y, por lo tanto, buenos indicadores para evaluar la calidad y continuidad del sistema poroso (Dörner y Dec, 2007). Tanto Ka como Ks de un suelo son parámetros que describen la funcionalidad de su sistema poroso, por lo tanto, nos permiten registrar el efecto de la intensidad de manejo del suelo sobre su calidad física, p.ej. los efectos del tipo de labranza y presión de pastoreo (Ellies et al., 1997; Dörner y Horn, 2006). Esto último cobra importancia, ya que para revertir los procesos de degradación de suelos bajo pradera naturalizadas degradadas existen distintas opciones que involucran distintos niveles de intervención: i) labrar el suelo y sembrar las especies pratenses deseadas, ii) incorporar al sistema las especies deseadas mediante una regeneración y iii) mejorar la productividad de una pradera a través de manejo y fertilización (Balocchi, 2007).

Según Ellies (1999) un manejo sustentable de praderas debería mejorar la estructura del suelo, aumentar la porosidad gruesa, mejorar la capacidad de arraigamiento y disminuir la resistencia a la penetración, tal que aumenta el almacenamiento de agua en el sue- lo quedando disponible para las plantas. Lo anterior, sin embargo, será dependiente de la forma en que se mejora la pradera. En ese contexto, el objetivo de este trabajo es determinar el efecto de tres estrategias de mejoramiento de praderas degradadas bajo pastoreos intensivos de producción de leche sobre propiedades hidráulicas del suelo.

\section{MATERIAL Y MÉTODO}

\section{Condiciones edafoclimáticas del sector de estudio}

Este ensayo fue realizado en la Estación Experimental Agropecuaria Austral (EEAA) ubicada a 6 kilómetros de la ciudad de Valdivia, sobre un suelo derivado de cenizas volcánicas de la Serie Valdivia, clasificado como Duric Hapludand, con pendientes dominantes de 3 a $8 \%$ y sectores ligeramente ondulados de 2 a $5 \%$ (CIREN, 2003). Estos suelos presentan altos contenidos de materia orgánica en horizontes superficiales (Zunino y Borie, 1985), alta estabilidad al agua y resistencia mecánica (Ellies et al., 1997). Por otro lado, se caracterizan por su alta capacidad para fijar fósforo (P) (Espinoza, 1991), baja densidad aparente, alta porosidad y una fuerte microagregación (Seguel y Orellana, 2008).

La temperatura promedio anual es de $12{ }^{\circ} \mathrm{C}$ y las precipitaciones pueden superar los $2.000 \mathrm{~mm}$ en el año (Huber, 1970) con una clara concentración de las mismas en invierno.

\section{Descripción de los tratamientos y criterios de pastoreo aplicados}

El ensayo ocupó una superficie total de $4.800 \mathrm{~m}^{2}$, formado por 12 (4 tratamientos con 3 repeticiones) parcelas dispuestas al azar con una dimensión de 20 $\mathrm{m} \times 20 \mathrm{~m}$ cada una $\left(400 \mathrm{~m}^{2}\right)$. Los tratamientos utilizados, fueron pradera degradada sin manejo (T1, DsF); pradera degradada con aplicación de fertilizantes (T2, DcF); pradera sembrada de Lolium perenne L. (Lp) y Trifolium repens $\mathrm{L}$. (Tr) con aplicación de fertilizantes (T3, ScF) y pradera sembrada mixta de L. perenne (Lp), T. repens (Tr), Dactylis glomerata L. (Dg), Bromus valdivianus (Bv) y Holcus lanatus (Hl), con aplicación de fertilizantes (T4, McF).

Para la fertilización de las praderas, se colectaron muestras de suelo de 0 a $20 \mathrm{~cm}$ de profundidad para determinar la caracterización química inicial del sitio del ensayo (Cuadro 1) mediante la metodología descrita por Sadzawka et al. (2006). Los tratamientos en las praderas naturalizadas y praderas sembradas en septiembre de 2006 fueron fertilizadas con dosis equivalente a: $40 \mathrm{~kg} \mathrm{~N} \mathrm{ha}^{-1}$ en forma de Nitromag; $120 \mathrm{~kg} \mathrm{P}_{2} \mathrm{O}_{5}$ $\mathrm{ha}^{-1}$ como Superfosfato Triple; $100 \mathrm{~kg} \mathrm{~K}_{2} \mathrm{O} \mathrm{ha}{ }^{-1}$ como Sulfato de Potasio y $2.000 \mathrm{~kg} \mathrm{Cal} \mathrm{ha}^{-1}$ como Cal Dolomítica. Adicionalmente, los tratamientos fueron fertiliza- 
Cuadro 1. Análisis de fertilidad de suelo del sitio experimental.

Table 1. Soil fertility analysis of the experimental site.

\begin{tabular}{lcc}
\hline Análisis & Unidad & Resultado \\
\hline $\mathrm{pH}$ en agua & $(1: 2,5)$ & 5,70 \\
$\mathrm{pH}$ en $\mathrm{CaCl}_{2}$ & $(1: 2,5)$ & 4,90 \\
Materia orgánica & $(\%)$ & 13,90 \\
$\mathrm{~N}-$ mineral $\left(\mathrm{N}-\mathrm{NO}_{3}-\mathrm{NH}_{4}\right)$ & $\left(\mathrm{mg} \mathrm{kg}^{-1}\right)$ & 16,80 \\
Fósforo Olsen & $\left(\mathrm{mg} \mathrm{kg}^{-1}\right)$ & 11,20 \\
Potasio intercambiable & $\left(\mathrm{mg} \mathrm{kg}^{-1}\right)$ & 142,00 \\
Sodio intercambiable & $\left(\mathrm{cmol}_{+} \mathrm{kg}^{-1}\right)$ & 0,08 \\
Calcio intercambiable & $\left(\mathrm{cmol}_{+} \mathrm{kg}^{-1}\right)$ & 2,36 \\
Magnesio intercambiable & $\left(\mathrm{cmol}_{+} \mathrm{kg}^{-1}\right)$ & 0,69 \\
Suma de bases & $\left(\mathrm{cmol}_{+} \mathrm{kg}^{-1}\right)$ & 3,49 \\
Aluminio intercambiable & $\left(\mathrm{cmol}_{+} \mathrm{kg}^{-1}\right)$ & 0,85 \\
CICE & $\left(\mathrm{cmol}_{+} \mathrm{kg}^{-1}\right)$ & 4,34 \\
\hline Saturación de Aluminio & $(\%)$ & 19,6 \\
\hline
\end{tabular}

dos con $25 \mathrm{~kg} \mathrm{~N} \mathrm{ha}^{-1}$ en diciembre de 2006 y febrero de 2007, y con $30 \mathrm{~kg} \mathrm{~N} \mathrm{ha}^{-1}$ en abril y julio de 2007.

Como criterio de pastoreo se siguió el nivel de producción de biomasa según estación. Para la estación de primavera, verano y otoño el criterio de entrada para pastorear fue de $2.400 \mathrm{~kg} \mathrm{MS} \mathrm{ha}^{-1}$ y de salida de 1.400 $\mathrm{kg} \mathrm{MS} \mathrm{ha-1}$. Para invierno, el criterio de entrada fue de $2.000 \mathrm{~kg} \mathrm{MS} \mathrm{ha}^{-1}$ y salida $1.200 \mathrm{~kg} \mathrm{MS} \mathrm{ha}^{-1}$. Para determinar la biomasa de entrada y salida de cada pastoreo, se utilizó el plato medidor de disponibilidad de forraje (Teuber et al., 2007). El pastoreo fue realizado por 14 vacas (2 $\left.\mathrm{UA} \mathrm{ha}^{-1} \mathrm{año}^{-1}\right)$ que ingresaban a las parcelas, las que eran retiradas cuando el residuo se encontraba a una altura de $5 \mathrm{~cm}$. Cuando no se alcanzaba la disponibilidad de entrada las vacas eran reingresadas cuando la ballica se encontraba en un estado de desarrollo de tercera hoja, o hubiera pasado un máximo de 60 días.

\section{Recolección de muestras de suelo y análisis de laboratorio}

Se recolectaron muestras no disturbadas de suelo en los primeros $5 \mathrm{~cm}$ de profundidad en cilindros metálicos de $230 \mathrm{~cm}^{3}(\mathrm{~h}=5,6 \mathrm{~cm}$ y $\phi=7,2 \mathrm{~cm})$. El suelo en los cilindros fue cubierto en los extremos y guardados en cajas herméticas para evitar evaporación y daño mecánicos durante el transporte. Adicionalmente, se recolectaron agregados de suelo y suelo disturbado para análisis fisicoquímicos.

Con las muestras no disturbadas se determinó la curva de retención de agua. Las muestras de suelo fue- ron saturadas por ascenso capilar de agua por 24 horas. Luego, fueron equilibradas a potenciales mátricos de: $-10,-20,-30$ y $-60 \mathrm{hPa}$ en bandejas de arena y -330 hPa en ollas de presión (Hartge y Horn, 2009). La densidad aparente se determinó secando las muestras en un horno a $105^{\circ} \mathrm{C}$ por $24 \mathrm{~h}$. A partir de la curva de retención de agua se determinó la distribución de poros del suelo (Hartge y Horn, 2009).

La conductividad hidráulica saturada (Ks) se midió en un permeámetro de carga constante, realizando mediciones a 1-3-6-12-24-48 y 96 horas después de iniciado el flujo de agua. La Ks fue determinada según la ecuación de Darcy:

$$
K s=\frac{V}{A * t} * \frac{L}{h}=\frac{Q * L}{h * A}
$$

donde, Ks = conductividad hidráulica en fase saturada $\left(\log \mathrm{cm}\right.$ día $\left.^{-1}\right) ; \mathrm{V}=$ volumen $\left(\mathrm{cm}^{3}\right) ; \mathrm{t}=$ tiempo (s); $\mathrm{Q}=$ caudal de agua percolada $\left(\mathrm{cm} \mathrm{s}^{-1}\right) ; \mathrm{L}=$ largo del cilindro $(\mathrm{cm}) ; \mathrm{h}=$ carga hidráulica $(\mathrm{cm}) ; \mathrm{A}=$ área del cilindro $\left(\mathrm{cm}^{2}\right)$.

Una vez determinada Ks se calculó la variación de la conductividad hidráulica (vKs), la cual representa el porcentaje que disminuye Ks al mantener un flujo constante de agua en el suelo por $96 \mathrm{~h}$ (Ellies et al., 1997), esta variable se obtiene de la siguiente fórmula:

$$
v K s=100-\left[\left(\frac{\text { Ks final }}{\text { Ks inicial }}\right) * 100\right]
$$

donde, vKs = variación de la conductividad hidráulica (\%); $\mathrm{Ks}_{\text {final }}=$ conductividad hidráulica en fase saturada después de 96 horas de flujo ( $\log \mathrm{cm}_{\text {día }}{ }^{-1}$ ); $\mathrm{Ks}_{\text {inicial }}=$ conductividad hidráulica en fase saturada inicial (log $\mathrm{cm}$ día $^{-1}$ ). Una vez terminadas las mediciones, los cilindros fueron saturados nuevamente y equilibrados a tensiones de 10, 20, 30, 60 y $330 \mathrm{hPa}$ para realizar una nueva curva de retención de agua.

La conductividad de aire ( $\mathrm{Kl})$ se determinó a los potenciales mátricos de -60 y $-330 \mathrm{hPa}$ por medio del método de flujo constante en un permeámetro de aire, aplicando una diferencia de presión menor a $1 \mathrm{hPa}$ para permitir un flujo laminar ascendente de aire a través de la muestra de suelo hasta alcanzar un flujo constante (Dörner y Dec, 2007). De esta manera, Kl se determinó a través de la siguiente ecuación:

$$
K l=\rho l * g *\left(\frac{\Delta V * L}{\Delta T * \Delta p * A}\right)
$$

Donde $\mathrm{Kl}=$ conductividad de aire $\left(\mathrm{cm} \mathrm{s}^{-1}\right) ; \rho \mathrm{l}=$ densidad de aire $\left(\mathrm{kg} \mathrm{m}^{-3}\right) ; \mathrm{g}=$ aceleración de gravedad $\left(\mathrm{m} \mathrm{s}^{-2}\right) ; \Delta \mathrm{V}$ $=$ volumen de aire que fluye $\left(\mathrm{m}^{3}\right) ; \mathrm{L}=$ largo cilindro $(\mathrm{m})$; 
$\Delta \mathrm{t}=$ tiempo (s); $\Delta \mathrm{p}=$ diferencia de presión ( $\mathrm{hPa}) ; \mathrm{A}=$ área cilindro $\left(\mathrm{cm}^{2}\right)$.

La conductividad de aire $(\mathrm{Kl})$ se expresó en términos de permeabilidad de aire, mediante la siguiente ecuación:

$$
K a=K l * \frac{\eta}{\rho \mathrm{l} * g}
$$

donde $\mathrm{Ka}=$ permeabilidad de aire $\left(\mu \mathrm{m}^{2}\right) ; \mathrm{Kl}=$ conductividad de aire $\left(\mathrm{cm} \mathrm{s}^{-1}\right) ; \eta=$ viscosidad de aire $\left(\mathrm{g} \mathrm{s}^{-1} \mathrm{~cm}^{-1}\right)$; $\rho \mathrm{l}=$ densidad de aire $\left(\mathrm{kg} \mathrm{m}^{-3}\right) ; \mathrm{g}=$ aceleración de gra$\operatorname{vedad}\left(\mathrm{m} \mathrm{s}^{-2}\right)$.

La estabilidad de los agregados se midió por el método del tamizaje en seco y tamizaje en húmedo, expresándose en la variación del diámetro medio ponderado de los agregados (VDMA) (Hartge y Horn, 1992). La estabilidad estructural fue inferida por VDMA, mediante el tamizado de agregados de suelo con un diámetro equivalente menor a $20 \mathrm{~mm}$, secado al aire (aproximadamente a $25^{\circ} \mathrm{C}$ ) y sin raíces. El material se colocó en un set de tamices de 12,5 - 8,0 - 6,3 - 4,0 - 1,0 - 0,5 mm, el cual se agitó a una intensidad constante (60 rpm) por 10 minutos. Una vez realizado el tamizado, se procedió a pesar el suelo retenido en cada tamiz para posteriormente devolverlo y repetir el procedimiento, pero esta vez en agua. El VDMA se obtiene de la siguiente manera:

$$
V D M A=\frac{\sum\left(n_{i 1} * d_{i}\right)-\left(n_{i 2} * d_{i}\right)}{\sum n_{i 1}}
$$

VDMA = variación del diámetro medio de los agregados $(\mathrm{mm}) ; \mathrm{n}_{i 1}=$ fracción tamizado en seco (\%); $\mathrm{n}_{i 2}=$ fracción tamizado en húmedo (\%); $\mathrm{d}_{\mathrm{i}}=$ diámetro ponderado de los agregados $(\mathrm{mm})$.

El ángulo de contacto se determinó en suelo tamizado a $20 \mathrm{~mm}$ (microagregados). Luego de esto se midió el ángulo de contacto en un microscopio con lupa de campo de visión horizontal provisto de goniómetro, tal como lo explican Bachmann et al. (2000). Cada medición se repitió 6 veces.

\section{Análisis estadístico}

Para el análisis estadístico se utilizó el programa STATGRAPHICS PLUS 5.1; en un diseño de cuatro tratamientos, con una distribución en terreno de bloques completamente al azar. Además, se realizó un análisis de varianza (ANDEVA) para determinar el efecto de los tratamientos. En el caso que se comprobara el efecto de los tratamientos, se aplicó una prueba de Tukey para determinar diferencias en las medias $(\mathrm{p}<0,05)$.

\section{RESULTADOS}

\section{Efecto de las estrategias de mejoramiento de praderas sobre la producción de forraje}

Existe una evolución en la productividad de las praderas dependiendo del manejo, siendo la pradera sembrada ( $\mathrm{ScF}$ ) la que presentó la mayor tasa de crecimiento de la pradera a partir del mes de Julio y una mayor producción anual de forraje, seguida por la pradera degradada con adición de fertilizante y la pradera sembrada mixta. Sin embargo, la mayor disponibilidad de forraje ( $\mathrm{kg} \mathrm{MS} \mathrm{ha}^{-1}$ ) al mes de Julio fue de la pradera degradada con adición de fertilizante, seguida de pradera sembrada mixta y pradera sembrada (Cuadro 2).

Cuadro 2. Disponibilidad de forraje de pre-pastoreo, producción anual de materia seca y tasa de crecimiento de la pradera para cada tratamiento.

\begin{tabular}{|c|c|c|c|}
\hline \multirow{3}{*}{ Pradera } & D.F.* & P.A.F & \multirow{3}{*}{$\begin{array}{c}\text { T.C.P } \\
\left(\text { kg MS ha }^{-1} \text { día }^{-1}\right) \\
\text { Julio } 2008\end{array}$} \\
\hline & \multicolumn{2}{|c|}{$\left(\mathrm{kg} \mathrm{MS} \mathrm{ha}^{-1}\right)$} & \\
\hline & Julio 2008 & Sept. 2007 - Sept. 2008 & \\
\hline Trat. 1 (DsF) & $1.571 \pm 128 \mathrm{a}$ & $3.554 \mathrm{a}$ & $2,3 \pm 0,9 \mathrm{a}$ \\
\hline Trat. 2 (DcF) & $2.473 \pm 190 \mathrm{~b}$ & $6.677 \mathrm{~b}$ & $6,1 \pm 0,1 b$ \\
\hline Trat. 3 (ScF) & $1.639 \pm 121 \mathrm{a}$ & $7.500 \mathrm{~b}$ & $8,6 \pm 0,7 c$ \\
\hline Trat. 4 (McF) & $2.416 \pm 245 b$ & $6.634 \mathrm{~b}$ & $5,9 \pm 0,6 b$ \\
\hline
\end{tabular}

Table 2. Forage availability of pre-grazing, annual dry matter production and pasture growth rate for each treatment.

*D.F. $=$ Disponibilidad de forraje; P.A.F. $=$ Producción anual de forraje; T.C.P. $=$ Tasa de crecimiento de pradera; DsF $=$ Degradada $($ testigo $) ;$ $\mathrm{DcF}=$ Degradada + fertilizante $; \mathrm{ScF}=$ Sembrada + fertilizante; $\mathrm{McF}=$ Mixta + fertilizante. Valores promedio \pm error estándar $(\mathrm{n}=15)$. En columnas, resultados seguidos con la misma letra no difieren significativamente entre sí, según prueba de Tukey $(\mathrm{p}<0,05)$. 
Efecto de las estrategias de mejoramiento de praderas sobre el sistema poroso y su estabilidad

La densidad aparente del suelo y el espacio poroso total, no presentaron variaciones entre los tratamientos evaluados (Cuadro 3). Sin embargo, se presentaron diferencias significativas en el volumen de poros de drenaje rápido (PDR, o equivalente a la capacidad de aire del suelo) entre los distintos tratamientos, donde el mayor valor lo presenta el DcF. Los poros de drenaje lento (PDL), poros de agua útil (PAU) e inútil (PAI) no presentaron diferencias significativas entre los manejos aplicados.

La siembra de praderas no provocó ningún cambio estadísticamente significativo en el sistema poroso durante el período de evaluación, tal que, de las distintas estrategias de mejoramiento de praderas aplicadas, sólo se observaron cambios en el sistema poroso del suelo con el DcF. Los valores obtenidos de la VDMA variaron entre $0,23(\mathrm{DcF})$ y $0,27(\mathrm{ScF}) \mathrm{mm}$, siendo este último estadísticamente distinto respecto a los otros tratamientos de pradera degradada y pradera sembrada mixta (Cuadro 4).

La conductividad hidráulica en fase saturada a través del tiempo no presentó diferencias estadísticas significativas $(p>0,05)$ entre las distintas estrategias de mejoramiento de praderas implementadas (Figura 1). Además, la Ks muestra una asociación directa $(\mathrm{p} \leq 0,10)$ con la cantidad de poros de drenaje rápido $\left(\mathrm{R}^{2}=0,789\right)$ y una asociación mayor con la porosidad efectiva del suelo (PDR+PDL) en los distintos tratamientos evaluados $\left(R^{2}=0,976\right)$ (Figura 2 ).

Cuadro 3.Densidad aparente (Dap) y distribución del espacio poroso (EP).

Table 3. Bulk density (Dap) and distribution of the porous space (EP).

\begin{tabular}{|c|c|c|c|c|c|}
\hline \multirow[b]{2}{*}{$\begin{array}{l}\text { Parámetros } \\
\text { evaluados }\end{array}$} & \multirow[b]{2}{*}{$\begin{array}{c}\text { Diámetro } \\
(\mu \mathrm{m})\end{array}$} & \multicolumn{4}{|c|}{ Praderas } \\
\hline & & $\begin{array}{l}\text { Trat. } 1 \\
\text { (DsF)* }\end{array}$ & $\begin{array}{l}\text { Trat. } 2 \\
\text { (DcF) }\end{array}$ & $\begin{array}{c}\text { Trat. } 3 \\
\text { (ScF) }\end{array}$ & $\begin{array}{l}\text { Trat. } 4 \\
\text { (McF) }\end{array}$ \\
\hline $\operatorname{Dap}\left(\mathrm{g} \mathrm{cm}^{-3}\right)$ & & $0,75 \mathrm{a}$ & $0,74 \mathrm{a}$ & $0,73 \mathrm{a}$ & $0,75 \mathrm{a}$ \\
\hline EP total (\% bv) & & $67,6 \pm 0,4 \mathrm{a}$ & $67,9 \pm 0,5 \mathrm{a}$ & $68,3 \pm 0,3 \mathrm{a}$ & $67,5 \pm 0,5 \mathrm{a}$ \\
\hline PDR (\% bv) & $>50$ & $10,6 \pm 0,7 \mathrm{a}$ & $16,4 \pm 0,9 b$ & $11,9 \pm 0,5 \mathrm{a}$ & $13,3 \pm 0,8 a$ \\
\hline PDL (\% bv) & $50-10$ & $12,9 \pm 0,3 \mathrm{a}$ & $10,3 \pm 0,4 \mathrm{a}$ & $13,8 \pm 0,4 \mathrm{a}$ & $11,1 \pm 0,4 \mathrm{a}$ \\
\hline PAU (\% bv) & $10-0,2$ & $17,5 \pm 0,5 \mathrm{a}$ & $16,9 \pm 0,7 \mathrm{a}$ & $17,4 \pm 0,7 \mathrm{a}$ & $17,8 \pm 0,6 a$ \\
\hline PAI (\% bv) & $<0,2$ & $25,2 \pm 0,3 a$ & $24,3 \pm 0,2 \mathrm{a}$ & $26,6 \pm 0,2 \mathrm{a}$ & $25,3 \pm 0,1 \mathrm{a}$ \\
\hline
\end{tabular}

*DsF = Degradada (testigo); DcF = Degradada + fertilizante; $\mathrm{ScF}=$ Sembrada + fertilizante; $\mathrm{McF}=$ Mixta + fertilizante. $\mathrm{PDR}(>50 \mu \mathrm{m})=$ Poros de drenaje rápido; PDL $(50-10 \mu \mathrm{m})=$ Poros de drenaje lento; PAU $(10-0,2 \mu \mathrm{m})=$ Poros de agua útil; PAI $(<0,2 \mu \mathrm{m})=$ Poros de agua inútil. Valores promedio \pm error estándar $(\mathrm{n}=15)$. En filas, resultados seguidos con la misma letra no difieren significativamente entre sí, según prueba de Tukey $(\mathrm{p}<0,05)$.

Cuadro 4. Parámetros físicos evaluados en los distintos tratamientos.

Table 4. Physical parameters evaluated in the different treatments.

\begin{tabular}{lcccc}
\hline & \multicolumn{3}{c}{ Praderas } \\
\hline $\begin{array}{l}\text { Parámetros } \\
\text { evaluados }\end{array}$ & $\begin{array}{c}\text { Trat. 1 } \\
\text { (DsF) }\end{array}$ & $\begin{array}{c}\text { Trat. } 2 \\
\text { (DcF) }\end{array}$ & $\begin{array}{c}\text { Trat. 3 } \\
\text { (ScF) }\end{array}$ & $\begin{array}{c}\text { Trat. 4 } \\
\text { (McF) }\end{array}$ \\
\hline vKs (\%) & $18,5 \pm 2,8 \mathrm{a}$ & $19,8 \pm 0,8 \mathrm{a}$ & $17,8 \pm 1,7 \mathrm{a}$ & $19,5 \pm 3,1 \mathrm{a}$ \\
VMDA (mm) & $0,24 \pm 0,0 \mathrm{~b}$ & $0,23 \pm 0,0 \mathrm{~b}$ & $0,27 \pm 0,0 \mathrm{a}$ & $0,24 \pm 0,0 \mathrm{~b}$ \\
Materia Orgánica (\%) & $15,4 \pm 0,2 \mathrm{a}$ & $15,5 \pm 0,3 \mathrm{a}$ & $14,9 \pm 0,4 \mathrm{a}$ & $15,8 \pm 0,4 \mathrm{a}$ \\
Angulo de contacto $\left(^{\circ}\right)$ & $88,1 \pm 0,7 \mathrm{a}$ & $89,9 \pm 0,8 \mathrm{a}$ & $87,8 \pm 0,5 \mathrm{a}$ & $90,1 \pm 0,3 \mathrm{a}$ \\
\hline
\end{tabular}

${ }^{*}$ DsF = Degradada (testigo) DcF = Degradada + fertilizante; $\mathrm{ScF}=$ Sembrada + fertilizante; $\mathrm{McF}=$ Mixta + fertilizante. vKs $=$ Variación de la conductividad hidráulica en fase saturada; $\mathrm{VMDA}=$ Variación del diámetro medio de los agregados (VDMA). Valores promedio \pm error estándar $(n=3)$. En filas, resultados seguidos con la misma letra no difieren significativamente entre sí, según prueba de Tukey $(\mathrm{p}<0,05)$. 
Los parámetros evaluados de vKs, contenido de materia orgánica y repelencia al agua (ángulo de contacto) no presentaron diferencias estadísticamente significativas ( $p>0,05$ ) en los tratamientos evaluados (Cuadro 4). Probablemente, el poco tiempo transcurrido desde la aplicación de las distintas estrategias de mejoramiento de praderas en este estudio no permite una diferenciación significativa en sus características físicas.

Los valores de permeabilidad de aire (Ka) evaluados para cada tratamiento a dos tensiones mátricas,

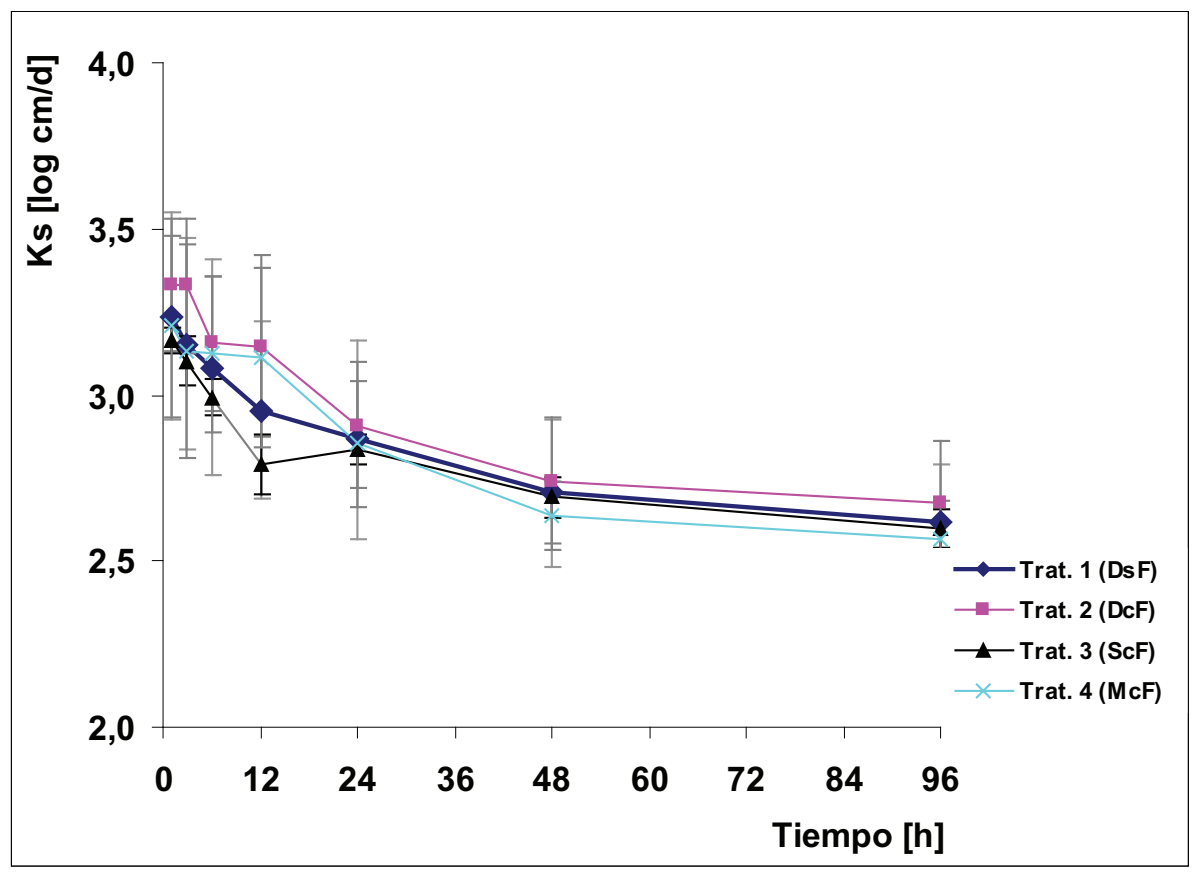

Figura 1. Conductividad hidráulica en fase saturada (Ks) para los distintos tratamientos. DsF = Degradada (testigo); DcF = Degradada + fertilizante; $\mathrm{ScF}=$ Sembrada + fertilizante; $\mathrm{McF}=$ Mixta + fertilizante. Valores promedio \pm error estándar $(\mathrm{n}=18)$.

Figure 1. Hydraulic saturated conductivity (Ks) for the different treatments. DsF = Degraded (Control); DcF = Degraded + fertilizer; $\mathrm{ScF}=$ Sown + fertilizer; $\mathrm{McF}=$ Mixed + fertilizer. Average values \pm standard error $(\mathrm{n}=18)$.

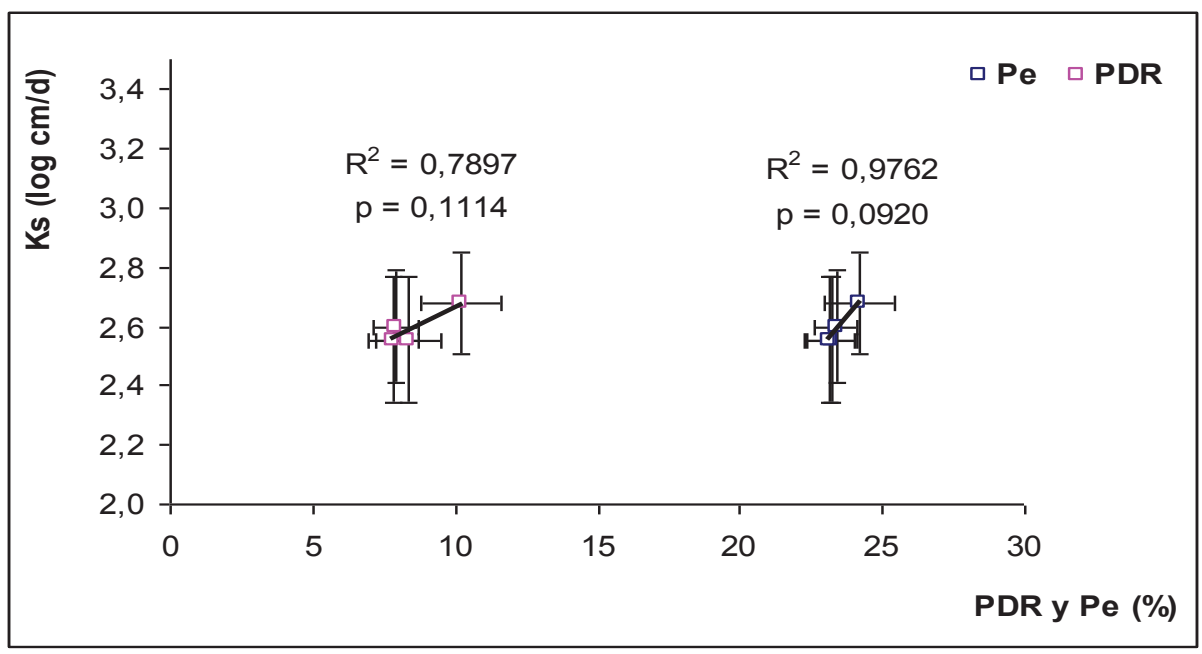

Figura 2. Relación entre la conductividad hidráulica en fase saturada (Ks) con los poros de drenaje rápido (PDR) y la porosidad efectiva (Pe) para los distintos tratamientos. *Valores promedio \pm error estándar $(\mathrm{n}=15)$.

Figure 2. Relationship between hydraulic saturated conductivity (Ks) with wide coarse pores (PDR) and the effective porosity $(\mathrm{Pe})$ for the different treatments. * Average values \pm standard error $(\mathrm{n}=15)$. 
mostraron que la mayor porosidad gruesa $(>10 \mu \mathrm{m})$ fue presentada por el DcF, aunque no significativamente diferente (Figura 3).

Los resultados obtenidos de Ka señalan claramente la dependencia que existe entre esta variable y la porosidad, ya que la pradera degradada (control) presenta el menor valor de $\mathrm{Ka}$, mientras que el tratamiento 2 (DcF) presentó una mayor cantidad de poros gruesos y un mayor valor de Ka $\left(\sim 2 \log \mu \mathrm{m}^{2}\right)$.

\section{DISCUSIÓN}

La aplicación de fertilizante en la pradera degradada permitió aumentar la producción de forraje e incrementar la capacidad de aire del suelo, probablemente por medio del incremento de la actividad biológica (formación de raíces, aumento de bioporos), lo que se traduce finalmente en mejores condiciones para el desarrollo vegetal. El manejo deficiente de praderas afecta la estructura del suelo, influyendo directamente en su sistema poroso, en la economía del agua, aire y el transporte de calor (Hartge y Horn, 1991). Suelos con baja cantidad de poros gruesos y/o grado de estructuración son características de praderas degradadas no intervenidas (Ellies et al., 1997). Praderas degradadas por la labranza tradicional y el manejo intensivo genera una disminución en la porosidad gruesa (macroporosidad). Por el contrario, los PAI se mantuvieron invariables en- tre los distintos tratamientos ya que el manejo del suelo no afecta la distribución del tamaño de las partículas ni el contenido de arcilla, el cual está estrechamente relacionado con los PAI (Baver y Gardner, 1973; Kutilek et al., 2006). Valores normales de PDR bajo pradera, dependiendo del tiempo de uso y el manejo que se realice, corresponden al rango entre 9,3-15\% para un suelo trumao (Torres, 1994; Nissen et al., 2006), valores menores a $10 \%$, son limitantes para el crecimiento de las plantas y la aireación del suelo, característico de suelos degradados (Greenwood y McNamara, 1992). Por el contrario, valores PDR entre $23-34 \%$ indican suelos no intervenidos antrópicamente (bosque), los cuales presentan una mayor proporción de PDR que suelos utilizados en producciones agrícolas tradicionales (Torres, 1994; Nissen et al., 2006). Por lo tanto, el manejo y el tiempo transcurrido influirían en la modificación de la estructura del suelo (Alarcon et al., 2010).

Balocchi (2007) indica que praderas sembradas (Ballica/Trébol), a largo plazo, deberían obtener productividades similares a las obtenidas con praderas naturales fertilizadas, lo cual no ocurre en el mes de julio, pero sí en la producción anual de la pradera. La tasa de crecimiento de praderas es baja durante el período invernal, dado principalmente por las bajas temperaturas y el exceso de agua que precipita, siendo en general menores a $10 \mathrm{~kg} \mathrm{MS} \mathrm{ha-1}$ día $^{-1}$ (Alamos, 2004; Hott, 2007). Aunque no se determinó una relación esta-

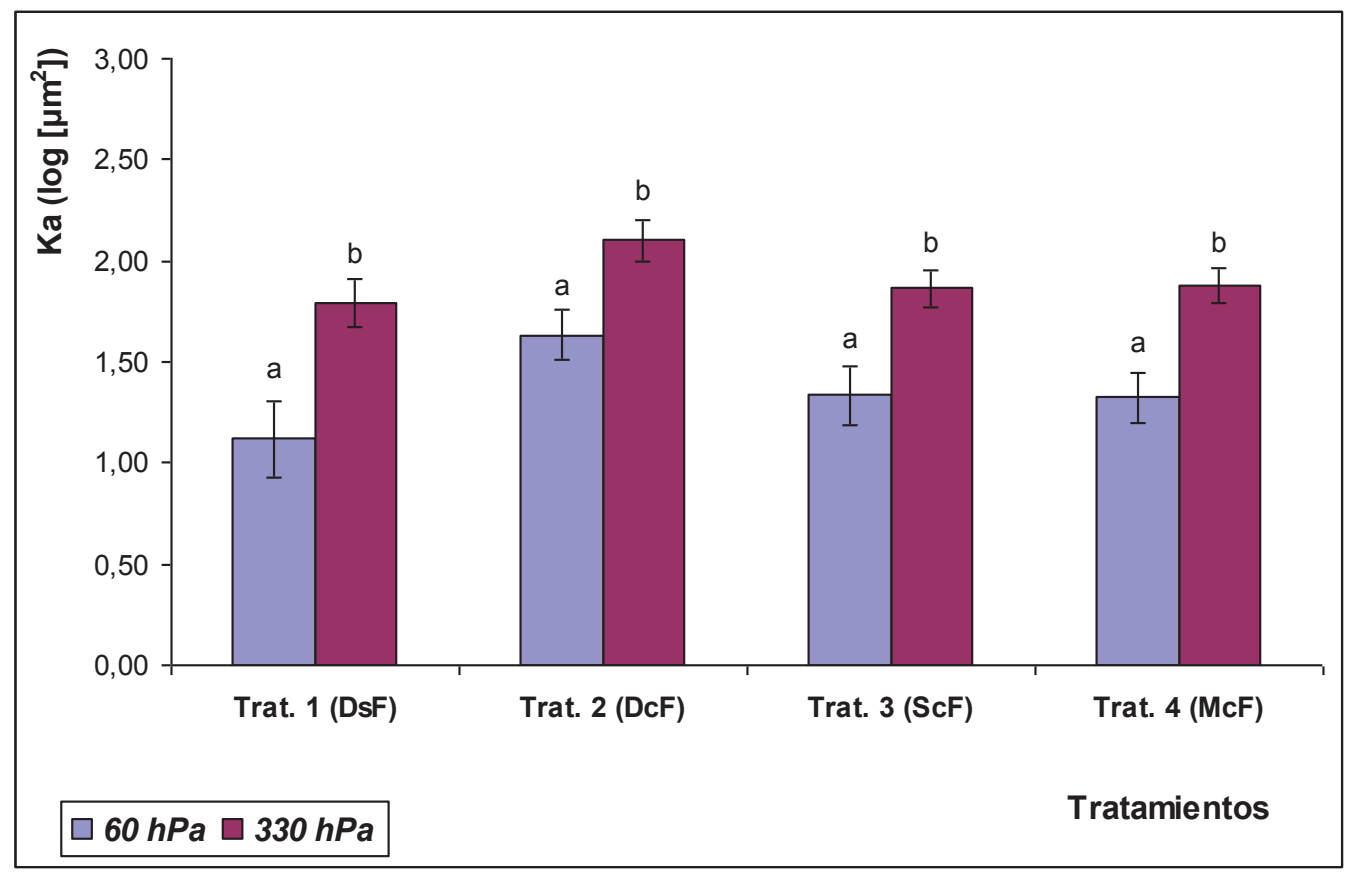

Figura 3. Permeabilidad de aire (Ka) de los tratamientos a distintas tensiones. Valores promedio \pm error estándar $(\mathrm{n}=6)$. En filas, resultados seguidos con la misma letra no difieren significativamente entre sí, según prueba de Tukey $(\mathrm{p}<0,05)$.

Figure 3. Air permeability (Ka) of treatments at different tensions. Average values \pm standard error $(n=6)$. Rows: results followed with the same letter do not differ significantly from each other, according to Tukey's test $(p<0.05)$. 
dísticamente significativa entre la tasa de crecimiento de la pradera y capacidad de aire por el corto periodo de evaluación del ensayo, si se puede observar una leve tendencia al incremento de la tasa de crecimiento cuando incrementan los poros de drenaje rápido del suelo. La tendencia señalada se debe a que la porosidad gruesa influye en el crecimiento vegetal al permitir el crecimiento más libre de las raíces y el consiguiente poblamiento del terreno con un número mayor de especies (Sommer, 1979).

\section{Estabilidad de la estructura del suelo}

Suelos sometidos a cultivos intensivos, tránsito de maquinaria y pisoteo animal, sufren el deterioro de la estructura, la cual se refleja en la disminución de la estabilidad de los agregados (Hartge y Ellies, 1990). Los suelos sometidos a manejos que conservan la estructura (bosque nativo, pradera artificial) en suelos de origen volcánico (Hapludands) presentan una alta estabilidad al agua o una baja variación del diámetro medio de sus poros, con lo cual, Ks no se altera sustancialmente (Ellies et al., 1997). Valores de vKs entre 40-50\% para suelos de origen ándico bajo pradera degradada y de $20-30 \%$ en la variación de la conductividad hidráulica bajo pradera artificial, son coincidentes con los determinados en este estudio, indicando que el manejo de praderas degradadas influye positivamente en la estructuración y continuidad del sistema poroso (Ellies et al., 1997; Medina, 2000).

La materia orgánica es considerada en la mantención de la estabilidad estructural, siendo un importante agente-ligado junto con las partículas minerales en la agregación de suelo (Baldock y Nelson, 1999). El efecto agregante se acentúa con la polaridad de estas uniones (Hamblin y Greenland, 1977). La estabilidad de los agregados al agua está muy relacionada con la capacidad o potencial que posean para humectarse, ya que para que un agregado se rompa, necesariamente debe haber una afinidad con el agua (Medina, 2000). En general, la mayor contribución al movimiento de agua en el suelo es atribuida a la porosidad gruesa; esta tendencia desaparece con el pasar del tiempo debido principalmente a oclusiones o aperturas que provocan cambios en la continuidad del sistema poroso (Ellies et al., 1997; Dörner y Horn, 2006). Suelos bajo pradera artificial alcanzan valores de Ks de 2,95 [log cm d-1] y en pradera degradada valores cercanos $2,70[\log \mathrm{cm}$ $\mathrm{d}^{-1}$, presentando una variación de Ks más alta, ya que presentarían una menor hidrofobia y menor resistencia de los agregados al agua (Ellies et al., 1996). En este estudio se determinaron valores superiores a los establecidos por Ellies et al. (1996) para praderas artificiales $\left(2,95 \log \mathrm{cm} \mathrm{d}^{-1}\right)$ y praderas degradadas $(2,70 \log$ $\left.\mathrm{cm} \mathrm{d}^{-1}\right)$, durante las primeras horas de medición $(3,10$ [ $\left.\log \mathrm{cm} \mathrm{d}^{-1}\right]$ aprox.), debido a la acción inicial de biopo- ros formados por el sistema radicular de las especies pratenses. La alta variabilidad de Ks se debe a la dependencia de esta propiedad con la estructura que presenta el suelo y está estrechamente relacionada con la distribución y continuidad del sistema poroso (Hartge y Horn, 1992; Dorner y Dec, 2007).

La porosidad efectiva (Pe), definida como el volumen de poros que contribuye principalmente en el movimiento de agua en fase saturada (PDR + PDL) se relaciona con Ks. De esta manera, Pe es responsable mayormente del movimiento de agua en fase saturada en el suelo, ya que, cuando se transmiten fluidos no solamente actúa la cantidad de poros, sino la continuidad que existe entre ellos, los cuales gobiernan el movimiento de agua (Dörner y Dec, 2007).

\section{Relación entre los parámetros que definen la estabilidad estructural del suelo frente al agua}

La resistencia a la humectación depende de la cantidad y el tipo de materia orgánica (Ellies et al., 1996). La repelencia al agua o hidrofobicidad de los suelos fue determinada en este caso a través del ángulo de contacto, el cual se correlaciona directamente con la cantidad de materia orgánica para todos los tratamientos $\left(R^{2}=0,692\right.$; $\mathrm{p}<0,05)$ y presenta una correlación inversa no significativa con VDMA $\left(R^{2}=0,656\right)$. La repelencia al agua o hidrofobicidad de los suelos, determinada en este caso a través del ángulo de contacto, está determinada por la interacción entre el contenido de materia orgánica y la textura del suelo (Cuevas, 2006). De esta manera, una moderada hidrofobia superficial del suelo es deseable, ya que en un suelo hidrófobo los agregados son más estables al agua, reduciendo la erosión al no dispersarse las partículas (Fabres, 2001). Por el contrario, una hidrofobia alta favorece el escurrimiento superficial y el arrastre de material provocando que el agua de lluvias estivales se aprovecha ineficientemente. Los resultados obtenidos, coinciden con estudios anteriores donde las mayores variaciones de Ks tienden a presentarse en aquellos suelos con una menor hidrofobia y menor resistencia de los agregados al agua (Ellies et al., 1996), ya que los tratamientos que presentan mayor hidrofobia (dada por la alta cantidad de materia orgánica y elevado ángulo de contacto) presentan agregados más estables a la dispersión y por lo tanto una baja VDMA. Esta relación sólo presenta una tendencia en las propiedades del suelo puesto que las diferencias entre las variables que componen la relación no son significativas debido al corto período de evaluación del ensayo en este estudio.

Por otro lado, la permeabilidad de aire aumenta exponencialmente conforme aumenta la fracción de poros disponibles para el movimiento de aire (Dörner y Horn, 2006). Esto coincide con lo apreciado para Ks, la cual aumenta a medida que la porosidad que influye sobre la conductividad aumenta. 
Efecto del tiempo de manejo sobre la estructura del suelo

Al evaluar el efecto de las estrategias de mejoramiento de praderas sobre el suelo se debe tener muy presente el tiempo transcurrido, ya que generalmente las modificaciones sobre las propiedades físicas del suelo causadas por el manejo sobre praderas se expresan a mediano y largo plazo (Bertol et al., 2000). Ellies et al. (1997) agregan que la magnitud de este cambio depende de la intensidad de uso del suelo y del tiempo de utilización de éste. Lo que explicaría en alguna medida la falta de relaciones estadísticamente significativas encontradas en esta investigación. Se ha establecido que las distintas propiedades del suelo varían de forma desigual en el tiempo. Las propiedades físicas relacionadas con la porosidad total del suelo, evaluada en sistemas conservacionistas, presentan un cambio significativo y estable en alrededor de 10 años, mientras que el efecto en la macroporosidad se presentó en 3 a 4 años, principalmente en los primeros $8 \mathrm{~cm}$ del suelo (Strudley et al., 2008). El manejo sustentable de praderas (labores culturales, siembra) mejora la estructuración del suelo, lo que se traduce principalmente en el aumento de la porosidad gruesa, mejor capacidad de arraigamiento, disminuye la resistencia a la penetración y posibilita o aumenta el almacenamiento de agua y el intercambio de fluidos en el suelo, por lo que se produce un menor escurrimiento superficial y por ende, disminuye la susceptibilidad a la erosión (Ellies, 1999). En el tiempo que llevan estas estrategias de mejoramiento ( 2 años), se aprecia un aumento efectivo en la productividad de la pradera, siendo las parcelas sembradas las que alcanzan una mayor producción, seguidas muy de cerca por la pradera degradada fertilizada. Sin embargo, el incremento de la productiva, no se traduce a las propiedades físicas del suelo, ya que sólo aumenta significativamente la porosidad gruesa del Trat. 2. Si se tiene en cuenta que la pradera naturalizada fertilizada obtiene producciones similares a las praderas sembradas a partir del tercer año, desde el punto de vista del mejoramiento físico del suelo en el corto plazo no se recomendaría la gran inversión en la que se debe incurrir al mejorar praderas a través de siembra, ya que la siembra de praderas sólo sería una alternativa para sectores en los que se realice rotación de cultivos (Siebald et al., 1983).

\section{CONCLUSIONES}

Las estrategias de mejoramiento de praderas estudiadas sólo aumentaron la capacidad del suelo para contener aire (macroporosidad), lo que tendría un efecto positivo en la producción de forraje. El resto de las variables evaluadas (porosidad total, Ks, Ka, materia orgánica y ángulo de contacto) no variaron como consecuencia de la utilización de distintas estrategias de mejoramiento. Sin embargo, se espera un real efecto en el mediano a largo plazo.

La fertilización de las praderas degradadas y la siembra de una pradera como alternativa de mejoramiento aumentan la productividad de esta, mientras sólo la fertilización de praderas (Trat. 2), dado por una mayor producción de biomasa radicular, aumenta la capacidad de aire del suelo, influyendo directamente en el transporte de agua y aire en el perfil. El manejo más degradante de la pradera aumenta la variación de la conductividad hidráulica, al ser sus agregados sensibles a la acción del agua. La mayor variación de la conductividad hidráulica tiende a presentarse en suelos menos repelentes al agua y que presentan una mayor VDMA.

\section{REFERENCIAS}

ALAMOS, A. 2004. Evaluación de la producción estacional de leche en la Décima Región Sur. Pontificia Universidad Católica de Chile, Facultad de Agronomía e Ingeniería Forestal. Departamento de Economía Agraria. Santiago.

ALARCÓN, C., DÖRNER, J., DEC, D., BALOCCHI, O., LÓPEZ, I. 2010. Efecto de dos intensidades de pastoreo sobre las propiedades hidráulicas de un andisol (Duric hapludand). Agro Sur 38(1): 30-41.

ALLENDE, P. 1993. Efecto de la compactación del suelo sobre el crecimiento radicular de especies pratenses. Tesis Lic. Agr. Valdivia. Universidad Austral de Chile. 40 p.

BACHMANN, J., HORTON, R., VAN DER PLOEG, R., WOCHE, S. 2000. Modified sessile drop for assessing soil - water contact angle of sandy soil. Soil Science Society of American Journal 64(2): 564 - 567.

BALDOCK, J., NELSON, P. 1999. Soil Organic Matter. In: Sumner, M.E. (Ed.), Handbook of Soil Science. CRC press, Boca Ratón, FL, pp. B-25-B-84.

BALOCCHI, 0. 2007. Mejoramiento de praderas naturales del sur de Chile. In: Hervé, M. (Ed.), Producción ovina: desde el suelo a la gestión, para profesionales y técnicos del área agropecuaria. Manuales FIA de apoyo a la formación de Recursos Humanos para la Innovación Agraria. Valdivia, pp. 13-21.

BAVER, L., GARDNER, W., GARDNER, W. 1973. Física de suelos. Unión tipográfica Hispanoamericana. México.

BERTOL, I., SCHICK, J., MASSARIOL, J.M., REIS, É.F.D., DILY, L. 2000. Propiedades físicas de um cambissolo húmico álico afetadas pelo manejo do solo. Ciência Rural 30(1): 91-95.

CENTRO DE INFORMACIÓN DE RECURSOS NATURALES (CIREN). 2003. Estudio agrológico X Región. Descripción de suelos, materiales y símbolos. Tomo II. Publicación $\mathrm{N}^{\circ} 123$, Santiago. 
CUEVAS, J., DÖRNER, J., ELLIES. A. 2004. Elementos de física y mecánica para evaluar la sustentabilidad de suelos agrícolas. Revista de la Ciencia del Suelo y Nutrición Vegetal 4:1-13.

DEC, D., DÖRNER, J., BALOCCHI, O. 2011. Temporal and spatial variability of structure dependent properties of a volcanic ash soil under pasture in southern Chile. Chilean Journal of Agricultural Research 71(2): 293-303.

DEC, D., DÖRNER, J., BALOCCHI, O., LÓPEZ, I. 2012. Temporal dynamics of hydraulic and mechanical properties of an Andosol under grazing. Soil and Tillage Research 125: 44-51.

DREWRY, J.J. 2006. Natural recovery of soil physical properties from treading damage of pastoral soils in New Zealand and Australia: A review. Agriculture, Ecosystems and Environment 114: 159-169.

DÖRNER, J., HORN, R. 2006. Anisotropy of pore functions in structuraI stagnic luvisols in the weichselian moraine region in Germany. Journal Plant Nutrition Soil Science 169: 212-220.

DÖRNER, J., DEC, D. 2007. La permeabilidad del aire y la conductividad hidráulica saturada como herramienta para la caracterización funcional de los poros del suelo. Revista de la Ciencia del Suelo y Nutrición Vegetal 7(2): 1-13.

ELLIES, A. 1994. Limitantes físicas del suelo en la producción de forrajes. In: Avances en producción animal. Producción Animal. Universidad Austral de Chile, Valdivia, pp. 23-38.

ELLIES, A., GREZ, R., RAMÍREZ, C. 1995. Potencial de humectación y estabilidad estructural de suelos sometido a diferentes manejos. Agricultura Técnica 55(3): 220-225.

ELLIES, A., GREZ, R., RAMÍREZ, C. 1996. Efecto de la materia orgánica sobre la capacidad de humectación y las propiedades estructurales de algunos Andosoles. Agro Sur 24(1): 48-60.

ELLIES, A., GREZ, R., RAMÍREZ, C. 1997. La conductividad hidráulica en fase saturada como herramienta para el diagnóstico de la estructura del suelo. Agro Sur 25(1): 51-56.

ELLIES, A. 1999. Manejo físico del suelo. Instituto de Investigaciones Agropecuarias (INIA). Series Remehue no. 71, Osorno, Chile, pp. 70-92.

ELLIES, A. 2000. Soil erosion and its control in Chile-An overview. Acta geológica hispánica 35(3): 279-284.

ESPINOZA, J. 1991. Efecto residual de fósforo en Andisoles. Revista Facultad de Agronomía (Maracay) 7: 39-47.

FABRES, N. 2001. Variación de la humectación de los suelos según intensidad de uso y propiedades de la materia orgánica. Tesis Lic. Agr. Valdivia, Universidad Austral de
Chile. 55 p.

GREENWOOD, K., MCKENZIE, B. 2001. Grazing effects on soil physical properties and the consequences for pastures: a review. Australian Journal of Experimental Agriculture 41(8): 1231-1250.

GREENWOOD, P., MCNAMARA, R. 1992. An analysis of the physical condition of two intensively grazed Southland soils. Proceedings of the New Zealand Grassland Association 54: 71-75.

HAMBLIN, A., GREENLAND, D. 1977. Effect of organic constituents and complexed metal ions on aggregate stability of some East Anglian soils. Journal of Soil Science 28: 410-416.

HARTGE, R., HORN, R. 2009. Die physikalische Untersuchung von Bóden. Praxis Messmethoden Auswertung. 4. vollst. Überarbeitete Auflage. Schweizerbart Vorlage, Stuttgart.

HARTGE, K., HORN, R. 1992. Die physikalische Untersuchung von Böden. Ferdinand Enke Verlag, 3. Auflage, Stuttgart.

HARTGE, K., HORN, R. 1991. Einführung in die dodenphysik. Enke. Stuttgart.

HARTGE, K., ELLIES, A. 1990. Changes in soil structure as caused by land use after clearing virgin forest. Zeitschrift für Pflanzenernährung und Bodenkunde 153: 389-393.

HILLEL, D. 1998. Environmental Soil Physics. Academic Press, San Diego, California.

HOTT, C. 2007. Tasa de crecimiento y composición nutricional de praderas permanentes en tres zonas agroecológicas de la zona Sur durante el verano, otoño e invierno. Tesis Lic. Agr. Valdivia, Universidad Austral de Chile, Facultad de Ciencias Agrarias. 57 p.

HUBER, A. 1970. Diez años de observaciones climatológicas en la Estación Teja, Valdivia (Chile). 1960 - 1969. Universidad Austral de Chile. Valdivia.

KUTILEK, M., JENELE, L., PANAYIOTOPOULOS, K. 2006. The influence of uniaxial compression upon pore size distribution in bi-model soils. Soil and Tillage Research 86: 27-37.

MEDINA, R. 2000. Efecto del manejo del suelo sobre la capacidad de humectación y la estabilidad estructural. Tesis Lic. Agr. Valdivia, Universidad Austral de Chile. 51 p.

NISSEN, J., QUIROZ, C., SEGUEL, O., MAC DONALD, R., ELLIES, A. 2006. Flujo hídrico no saturado en Andisoles. Revista de la Ciencia del Suelo y Nutrición Vegetal 6(1): 9-19.

SADZAWKA, A., CARRASCO, M., GREZ, R., MORA, M., FLORES, H., NEAMAN, A. 2006. Métodos de análisis recomendados para los suelos de Chile, Instituto de Investigaciones Agropecuarias, serie actas INIA № 34. Santiago, Chile. 
SEGUEL, O., ORELLANA, I. 2008. Relación entre las propiedades mecánicas de suelos y los procesos de génesis e intensidad de uso. Agro Sur 36(2): 34-44.

SHOJI, S., NANZYO, M., DAHLGREN, R. 1993. Volcanic Ash Soils Genesis, Properties and Utilization. Elsevier, Amsterdam.

SIEBALD, E., MATZNER, M., BECKER, F. 1983. Mejoramiento de praderas naturales del Llano Central de la Décima Región. Agricultura Técnica 43: 313-321.

STRUDLEY, M., GREEN, T., ASCOUGH, J. 2008. Tillage effects on soil hydraulic properties in space and time: state of the science. Soil and Tillage Research 99: 4-48.

TABOADA, M. 2007. Efectos del pisoteo y pastoreo animal sobre suelos en siembra directa. Cátedra de Fertilidad y fertilizantes. Facultad de Agronomía. Universidad de Buenos Aires, Argentina.

TEUBER, N. 1996. La pradera en el llano longitudinal de la X Región (Valdivia -Chiloé), In: Ruiz, I. (Ed.), Praderas para
Chile. Instituto de Investigaciones Agropecuarias, INIA, Ministerio de Agricultura. Santiago, Chile, pp. 536-544.

TEUBER, N., BALOCCHI, O., PARGA, J. 2007. Manejo del pastoreo. INIA Remehue, Universidad Austral de Chile, Universidad de la Frontera, Fundación para la Innovación Agraria. Osorno, Chile.

TORRES, E. 1994. Variación de la estructura del suelo por la intensidad y tiempo de uso. Tesis Lic. Agr. Valdivia. Universidad Austral de Chile. 45 p.

TORRES, A. 2006. Antecedentes de la producción de leche en el sur de Chile. In: Navarro, H., Siebald, E., Celis, S. (Eds.), Manual de producción de leche para pequeños y medianos productores. Gobierno de Chile, Instituto de Investigaciones Agropecuarias. Osorno, Chile.

ZUNINO, H., BORIE, F. 1985. Materia orgánica y procesos biológicos en suelos alofánicos. In: Tosso, J. (Ed.), Suelos volcánicos de Chile. Instituto de Investigaciones Agropecuarias (INIA), Santiago, pp. 341-425. 
